\title{
Relationship between glucose homeostatic disorders and testosterone in adult Nigerian women with polycystic ovary syndrome
}

\author{
Collins Amadi ${ }^{1 *}$, Kinikanwo I. Green ${ }^{2}$
}

\begin{abstract}
${ }^{1}$ Department of Chemical Pathology and Metabolic Medicine, ${ }^{2}$ Department of Obstetrics and Gynecology, University of Port Harcourt Teaching Hospital, Port Harcourt, Nigeria.
\end{abstract}

Received: 30 August 2018

Accepted: 28 September 2018

\author{
*Correspondence: \\ Dr. Collins Amadi, \\ E-mail: collins338@yahoo.com
}

Copyright: (C) the author(s), publisher and licensee Medip Academy. This is an open-access article distributed under the terms of the Creative Commons Attribution Non-Commercial License, which permits unrestricted non-commercial use, distribution, and reproduction in any medium, provided the original work is properly cited.

\begin{abstract}
Background: Polycystic ovary syndrome (PCOS) is associated with hyperandrogenemia which is hypothesized to induce glucose homeostastic disorders among women with the syndrome. This study was thereby set to investigate the status of glucose homeostatic disorders and their relationship with testosterone among women with PCOS in Port Harcourt, Nigeria.

Methods: This was a retrospective study of 224 women with PCOS who had visited a tertiary hospital in Nigeria over a 10 -year period. The laboratory characteristics of these patients were evaluated. Records on age, fasting plasma glucose (FPG) and serum total testosterone (TT) were collected and analyzed using Shapiro-Wilk, descriptive, chisquare, Fisher's exact, two-way analysis of variance, and Pearson's tests. A p-value $<0.05$ was considered significant. Results: Glucose homeostatic disorders were documented in $49.1 \%$ of the study population. Impaired fasting glucose (IFG) and diabetes mellitus (DM) was observed among $37.5 \%$ and $11.6 \%$ of the study population respectively. There was difference in the mean TT levels among those with normal FPG, IFG, and DM ( $<<0.001)$. Those with DM had higher TT levels $(2.47 \pm 0.83 \mathrm{nmol} / \mathrm{l})$ than those with IFG $(2.12 \pm 0.63 \mathrm{nmol} / \mathrm{l})$ and normal glucose homeostatic status $(1.63 \pm 0.47 \mathrm{nmol} / \mathrm{l})$. TT correlated positively with FPG among the overall study population $(\mathrm{r}=0.465 ; \mathrm{p}$-value $=<$ $0.001)$, among those with IFG $(r=0.591$; $p$-value $=0.004)$, and among those with DM $(r=0.404$; $p$-value $=0.012)$.

Conclusions: Glucose homeostatic disorders are common among PCOS patients in our environment, especially among those with higher testosterone levels. This warrants screening of all PCOS patients for glucose disorders as part of their management strategies.
\end{abstract}

Keywords: Diabetes mellitus, PCOS, Testosterone, Impaired fasting glucose

\section{INTRODUCTION}

Polycystic ovary syndrome (PCOS), first described in 1935 by Stein and Leventhal as a triad of hirsutism, amenorrhea and polycystic ovary morphology, is a heterogeneous and multifaceted disorder that affects about $5-10 \%$ of reproductive-aged women worldwide. ${ }^{1,2}$ The syndrome is primarily characterized by chronic ovulatory dysfunction, hyperandrogenism, and polycystic ovaries on ultrasound scan. ${ }^{3}$ Hyperandrogenism secondary to hyperandrogenemia (excess blood androgens) is the most characteristic hallmark of the syndrome. ${ }^{1-3}$ The etiopathogenesis of PCOS is unknown, however, several theories had been postulated as possible agents in the evolution of the syndrome. ${ }^{4}$ The involvement of some genetic factors acting in concert with certain environmental agents is currently the most prominent and widely accepted theory in the evolution of the syndrome. ${ }^{4,5}$ The clinical diagnosis of the syndrome is based on the National Institute of Health, Androgen Excess Society, and the Rotterdam criteria due to the complexity of its spectrum of clinical signs and symptoms. ${ }^{6-8}$

Initially, the syndrome was thought to be primarily a female reproductive dysfunction when it was first 
described in the literature by Stein and Leventhal, however, a large number of epidemiologic evidence exist which suggest that the syndrome is rather a multicomplex disorder with various gynecological, reproductive, endocrine and metabolic consequences associated with insulin resistance, hyperinsulinemia, glucose homeostatic disorders (impaired state and frank diabetes), hypertension, and lipid abnormalities with increased risk of adverse cardiovascular events. Insulin resistance is adjudged a well-recognized associated metabolic factor with various implications in the pathophysiology of PCOS. ${ }^{9}$

As a consequence of the insulin resistant state inherent in PCOS, numerous studies had documented the increased prevalence of glucose homeostatic disorders among women with PCOS compared to normal control population. ${ }^{10}$ Androgen excess, associated with the syndrome, is hypothesized to influence glucose homeostasis in patients with PCOS. ${ }^{11}$

Nevertheless, there is limited data on the status of these glucose homeostatic disorders and their relationship with testosterone among PCOS patients in our region. Therefore, this study was designed to investigate the pattern of glucose homeostatic disorders and their relationship with serum testosterone among adult women with PCOS in Port Harcourt, Nigeria.

The objectives of the present study were to determine the rate of glucose homeostatic disorders among the study population, to determine the pattern of glucose homeostatic disorders among the study population, to determine the relationship between testosterone and plasma glucose among the various glucose homeostatic disorders, and to compare the findings in this study with the literature.

\section{METHODS}

The study was undertaken in the Department of Chemical Pathology and Metabolic Medicine of one of the tertiary hospital care centers in Nigeria-University of Port Harcourt Teaching Hospital (UPTH), Port Harcourt, Nigeria. This tertiary hospital located in the heart of Niger Delta region of Nigeria is a major referral center for all the primary and secondary health centers in the region.

The Department of Chemical Pathology and Metabolic Medicine is one of the four departments of Pathology in the hospital which undertakes daily clinical chemistry analysis with an attached metabolic clinic where uncomplicated metabolic disorders are referred to from different units within the hospital.

The was a retrospective, descriptive, and cross-sectional study of adult women with PCOS who presented to the Department of Chemical Pathology and Metabolic Medicine of the University of Port Harcourt Teaching hospital (UPTH) from different sources within the hospital from 1st January 2008 to 31st December 2017. All patients had been diagnosed by the specialist gynecologist in UPTH based on the Rotterdam criteria which is defined as the presence of any two of the following three features of the syndrome ${ }^{8}$;

- Chronic oligo-ovulation/anovulation evidenced either biochemically (day 21-23 menstrual cycle serum progesterone of less $10 \mathrm{ng} / \mathrm{ml}$ or $32 \mathrm{nmol} / \mathrm{l}$ ) or clinically (oligo-menorrhea/amenorrhea).

- Hyperandrogenism evidenced either clinically (hirsutism, acne, and androgenic alopecia) or biochemically (total serum testosterone $>0.8 \mathrm{ng} / \mathrm{ml}$ or $2.8 \mathrm{nmol} / \mathrm{l})$.

- Polycystic ovaries on transvaginal ultrasound scan defined as the presence of twelve or more follicles measuring $2-9 \mathrm{~mm}$ in diameter and/or at least one enlarged ovary measuring more than $10 \mathrm{~cm}^{3}$.

\section{Inclusion criteria}

- Records of age, serum total testosterone and fasting plasma glucose concentrations of patients more than 18 years with any phenotype of PCOS.

\section{Exclusion criteria}

- Records of PCOS patients who are either pregnant, with established diabetes mellitus, with hyperprolactinemia, with established thyroid disorders or with any other androgen excess disorders including those with incomplete data.

During the period under study, fasting specimen of whole blood were collected from each patient by phlebotomy. All acquired specimen were emptied into fluoride oxalate and plain specimen collection bottles and subsequently processed accordingly (centrifugation and separation of supernatant) to obtain the plasma and serum samples for analysis.

Laboratory analysis for plasma glucose were all carried out through the glucose oxidase enzymatic method with same brands of laboratory reagents purchased from Randox Laboratory manufacturers, United Kingdom, while serum TT was analyzed via competitive enzyme immunoassay method with reagents sourced from Monoblind Incorporated, United States. ${ }^{12,13}$

To maintain and monitor analytical precision and accuracy, three levels of commercial control sera, sourced from the same reagent manufacturers, were interspersed in each run of the laboratory analysis.

\section{Statistical analysis}

All records from laboratory result sheets and case notes of each PCOS patient were collected, reviewed and entered into Statistical Package for Social Sciences 
(SPSS) version 20. Records collected were demographic data (age in years), clinical diagnosis (PCOS), and fasting plasma glucose concentration in $\mathrm{mmol} / \mathrm{l}$, and early follicular phase (day 3) serum total testosterone (TT) in nmol/l (Reference threshold $2.8 \mathrm{nmol} / \mathrm{l}$ ). Fasting plasma glucose levels were defined based on the expert recommendation of the American Diabetes Association 2003 criteria which entails the followings ${ }^{14}$ :

- Normal glucose homeostasis (fasting plasma glucose of less than $5.6 \mathrm{mmol} / \mathrm{l}$

- Impaired fasting plasma glucose (fasting plasma glucose between 5.6-6.9 mmol/l)

- Diabetes mellitus likely (fasting plasma glucose greater than $7.0 \mathrm{mmol} / \mathrm{l}$ )

Serum TT was stratified as normal $(<2.8 \mathrm{nmol} / \mathrm{l})$ or excess $(>2.8 \mathrm{nmol} / \mathrm{l})$.

Normality of the abstracted continuous data was first determined using Shapiro-Wilk statistical test and all skewed data were logarithmically transformed prior to analysis. Then descriptive statistics (mean \pm standard deviation and range), Chi-square test, Fisher's exact test for cells less than five, two-way analysis of variance (ANOVA) for comparison of mean value of more than two continuous normally distributed variables, and Pearson's correlation test to determine the relationship between two continuous normally distributed variables were all employed in the statistical analysis.

A p-value of $<0.05$ was considered statistically significant.

\section{RESULTS}

During the period of 1st January 2008 to December 2017, two hundred and sixty-six (266) patients with PCOS were referred from different sources of UPTH to the Department of Chemical Pathology and Metabolic Medicine for estimation of fasting plasma glucose and serum total testosterone. The data of 224 of these patients met the inclusion criteria of the present study and were eventually recruited.

Using Shapiro-Wilk test, the age variable was found to be normally distributed.

However, that of the fasting plasma glucose (Z-score = $+5.666 ; \mathrm{p}$-value $=0.001)$ and serum total testosterone $(\mathrm{Z}$ score $=+3.375 ;$ p-value $=0.003$ ) were not normally distributed.

Thereafter, the data of fasting plasma glucose and that of serum total testosterone were logarithmically transformed prior to subsequent statistical analysis.

Glucose homeostatic disorders were observed in 110 (49.1\%) of the study population while $114(50.9 \%)$ had normal glucose homeostatic status. Excess testosterone (hyperandrogenemia) was observed in $76(33.9 \%)$ of the study population while $148(66.1 \%)$ had normal serum testosterone levels. In table 1 , the mean \pm standard deviation and the range of the study population age, fasting plasma glucose concentrations and the serum total testosterone were $28.29 \pm 5.63$ years; range $18-42$ years, $5.70 \pm 1.24 \mathrm{mmol} / \mathrm{l}$; range $3.8-9.3 \mathrm{mmol} / \mathrm{l}$, and $1.91 \pm 0.63$ $\mathrm{nmol} / \mathrm{l}$; range $0.8-4.2 \mathrm{nmol} / \mathrm{l}$ respectively. Furthermore, the mean fasting plasma glucose concentration $(5.70 \pm 1.24 \mathrm{mmol} / \mathrm{l})$ of the entire study population was within the impaired fasting plasma glucose status as defined by the American Diabetes Association.

Table 1: Descriptive characteristics of study variables.

\begin{tabular}{|lcll|}
\hline Variable & n & \multicolumn{1}{c}{ Range } & Mean \pm SD \\
\hline Age (years) & 224 & $18-42$ & $28.29 \pm 5.63$ \\
\hline FPG (mmol/l) & 224 & $3.8-9.3$ & $5.70 \pm 1.24$ \\
\hline TT $(\mathrm{nmol} / \mathrm{l})$ & 224 & $0.8-4.2$ & $1.91 \pm 0.63$ \\
\hline
\end{tabular}

$\mathrm{SD}=$ Standard deviation; FPG = Fasting plasma glucose; $\mathrm{mmol} / \mathrm{l}=$ Millimole per liter; $\mathrm{n}=$ Frequency .

In table 2, the observed frequency of those with diabetes mellitus is $11.6 \%$ (26) while those with impaired fasting plasma glucose was $37.5 \%$ (84) among the study population. The majority $(50.9 \%)$ of the study population had normal glucose homeostatic status.

Table 2: Distribution of fasting plasma glucose groups based on ADA criteria.

\begin{tabular}{|c|c|c|c|c|}
\hline $\begin{array}{l}\text { ADA Classification } \\
\text { of FPG }\end{array}$ & $\mathbf{N}$ & $\%$ & $\gamma^{2}$ & p-value \\
\hline$<5.6 \mathrm{mmol} / \mathrm{l}$ (normal) & 114 & 50.9 & \multirow{3}{*}{53.607} & \multirow{3}{*}{$<0.001 *$} \\
\hline $\begin{array}{l}5.6-6.9 \mathrm{mmol} / 1 \\
\text { (impaired) }\end{array}$ & 84 & 37.5 & & \\
\hline$>7.0 \mathrm{mmol} / \mathrm{l}(\mathrm{DM})$ & 26 & 11.6 & & \\
\hline
\end{tabular}

In table 3, there were statistically significant difference in the mean serum total testosterone concentrations among those with normal glucose homeostatic status, impaired fasting plasma glucose, and those with established diabetes mellitus ( $\mathrm{p}$-value $<0.001)$.

Table 3: Comparison of serum testosterone between different strata of FPG groups.

\begin{tabular}{|c|c|c|c|}
\hline FPG stratum & $\begin{array}{l}\text { Serum TT } \\
(\text { nmol/I) } \\
\text { Mean } \pm \text { SD }\end{array}$ & $\mathbf{F}$ & p-value \\
\hline $\begin{array}{l}<5.6 \mathrm{mmol} / / \\
\text { (Normal) }\end{array}$ & $1.63 \pm 0.47$ & \multirow{3}{*}{33.740} & \multirow{3}{*}{$<0.001 *$} \\
\hline $\begin{array}{l}5.6-6.9 \mathrm{mmol} / 1 \\
\text { (Impaired) }\end{array}$ & $2.12 \pm 0.63$ & & \\
\hline$>7.0 \mathrm{mmol} /(\mathrm{DM})$ & $2.47 \pm 0.83$ & & \\
\hline
\end{tabular}


Moreover, the mean total serum testosterone concentrations was higher among the study group with impaired fasting plasma glucose and those with diabetes mellitus. However, those with diabetes mellitus had a higher mean serum total concentration $(2.47 \pm 0.83$ $\mathrm{nmol} / \mathrm{l}$ ) than those with the impaired fasting plasma glucose $(2.12 \pm 0.63 \mathrm{nmol} / \mathrm{l})$ and those with normal glucose homeostatic status $(1.63 \pm 0.47 \mathrm{nmol} / \mathrm{l})$.

In table 4, there were varying degrees of positive correlations between serum total testosterone and fasting plasma glucose among the overall study population, those with normal glucose homeostatic status, those with impaired fasting glucose status, and among those with diabetes mellitus.

Table 4: Correlation of TT and FPG among different study groups.

\begin{tabular}{|c|c|c|}
\hline \multirow[b]{2}{*}{ Study groups } & \multicolumn{2}{|c|}{ TT versus FPG } \\
\hline & $\begin{array}{l}\text { Correlation } \\
\text { coefficient (r) }\end{array}$ & p-value \\
\hline Overall study population & 0.465 & $<0.001 *$ \\
\hline $\begin{array}{l}<5.6 \mathrm{mmol} / \mathrm{l} \text { group } \\
\text { (normal) }\end{array}$ & 0.188 & 0.045 \\
\hline $\begin{array}{l}\text { 5.6-6.7 mmol/1 group } \\
\text { (Impaired) }\end{array}$ & 0.591 & $0.004 *$ \\
\hline$>7.0 \mathrm{mmol} / \mathrm{l}$ group $(\mathrm{DM})$ & 0.404 & $0.012 *$ \\
\hline
\end{tabular}

*Statistically significant; TT=Total testosterone; FPG=Fasting plasma glucose; $\mathrm{mmol} / \mathrm{l}=$ millimole per liter; $\mathrm{DM}=$ Diabetes mellitus

However, the correlations of the total serum testosterone and fasting plasma glucose was strong and statistically significant among the overall study population $(r=0.465$; p-value $=<0.001)$, among those with impaired fasting plasma status $(\mathrm{r}=0.591 ; \mathrm{p}$-value $=0.004)$, and among those with established diabetes mellitus $(r=0.404$; $\mathrm{p}$ value $=0.012$ ) .

\section{DISCUSSION}

Since the time it was first described, polycystic ovary syndrome (PCOS) had traditionally been characterized as a disorder of female reproductive physiology. ${ }^{1}$ However, over the decade, there has been an enormous appreciation that the syndrome is associated with increased risk of a variety of metabolic disturbances such obesity, insulin resistance, hyperinsulinemia, hypertension, nonalcoholic fatty liver diseases, and dyslipidemia.

Glucose homeostatic disorders (impaired and frank diabetes) seem to cluster among patients with PCOS. ${ }^{3,15}$ Several rates of these glucose disorders have been documented by different authors using different criteria in the literature, but the rates ranged from $30 \%-40 \%{ }^{16}$

In this present study, we had evaluated these disorders of glucose metabolism using fasting plasma glucose and observed a $49.1 \%$ rate of these glucose homeostatic abnormalities among the present study population which is higher than the rates reported in the literature. ${ }^{16}$ Based on the definition by the American Diabetes Association (ADA 2003), the rate of the specific glucose homeostatic abnormalities observed among present study population was $37.5 \%$ of impaired fasting glucose (IFG) and $11.6 \%$ of diabetes mellitus (DM). Similar studies had reported lower rates of impaired fasting plasma glucose and diabetes mellitus among adult PCOS patients using the ADA 2003 criteria as used in this study. ${ }^{17-19}$

Lerchbaum and colleagues reported $12.8 \%$ and $1.5 \%$ rates of IFG and DM respectively, Barcellos and colleagues reported $15.3 \%$ and $1.2 \%$ rates of IFG and DM respectively while Erhmann and colleagues reported a $9 \%$ and $6 \%$ of IFG and DM respectively. ${ }^{17-19}$

The higher rates of IFG and DM documented in this study compared to these similar previous cited studies suggest increased prevalence of glucose homeostatic disorders among women with PCOS in our environment. ${ }^{17-19}$

Besides, these reported rates of IGT and DM observed in this study is significantly higher than the rate documented among healthy Nigerian without PCOS. ${ }^{20-21}$

Insulin resistance, $\beta$-cell dysfunction, and obesity have all been characterized as major factors involved in the abnormal glucose homeostatic status in patients with PCOS. ${ }^{22-23}$

This abnormal glucose homeostasis had been reported to be more pronounced in women with excess testosterone than their counterpart PCOS patients with normal testosterone status, thus establishing excess testosterone as another factor involved in these abnormalities of glucose metabolism. ${ }^{23}$

This is evident in this study where we had noted higher testosterone levels among PCOS patients with IFG and DM than those with normal glucose homeostasis, supported by the positive correlations of testosterone and glucose among patients with IFG and DM.

Excess testosterone in patients with PCOS, in addition to its reported involvement in insulin resistance and $\beta$-cell dysfunction, enhances central adiposity that initiates insulin resistance which predisposes women with PCOS to these abnormal glucose metabolism. ${ }^{22-23}$

The present study has some limitation that warrants mentioning. Firstly, it is a retrospective hospital-based study carried out in a single center. So, its findings might not be representative of the entire population in our region.

Secondly, fasting plasma glucose as an assessment tool for glucose disorders in PCOS has been documented to exhibit low sensitivity leading to the underestimation of 
the true degree of these glucose homeostatic disorders in patients with PCOS. However, the American Diabetes Association recommend the use of fasting plasma glucose test as a screening tool for these disorders. ${ }^{12}$

Thirdly, the only androgen accounted for in this study is testosterone which is one of the androgens involved in the pathophysiology of PCOS. The other androgens, such androstenedione and dehydroepiandrosterone sulphate, also contribute to the hyperandrogenism but are not routinely recommended in PCOS investigations. ${ }^{24}$

The free testosterone and sex hormone binding globulin were not accounted for in this study which may or may not have influenced the outcome of this study if considered.

\section{CONCLUSION}

Evidence from this study demonstrates high level of glucose homeostatic abnormalities among adult Nigerian women with PCOS. We recommend screening for these glucose abnormalities among these patients with PCOS as part of their management strategies.

\section{ACKNOWLEDGMENTS}

Authors would like to thank all the laboratory staff in the Department of Chemical Pathology and Metabolic of UPTH for their assistance during the conduct of this study.

\section{Funding: No funding sources}

Conflict of interest: None declared

Ethical approval: The study was approved by the Institutional Ethics Committee

\section{REFERENCES}

1. Stein IF, Leventhal ML. Amenorrhoea associated with bilateral polycystic ovaries. Am J Obstet Gynecol. 1935;29:181-91.

2. Lavie O. Benign disorders of the ovaries and oviducts. In: Alan HD, Lauren N, Ashley SR, editors. Current Obstetrics and Gynaecologic Diagnosis and Treatment. $11^{\text {th }}$ ed. New York: Lange Medical Publication; 2013. pp. 661-70.

3. Moharaj S, Amod A. Polycystic ovary syndrome. J Endocrinol Metab S Afr. 2009;14(2):86-95.

4. Goodarz MO, Dumesic DA, Chazenbalk G, Azziz R. Polycystic ovary syndrome: etiology, pathogenesis and diagnosis. Nat Rev Endocrinol. 2011; 7(4): 21931.

5. Joe-Kechebelu NN, Mbamara SU, Ikechebelu JI. Familial trend in polycystic ovarian syndrome. Ann Afr Med. 2013;12(3):132-4.

6. Zawadic JK, Danif A. Diagnostic criteria for polycystic ovarian syndrome: towards a rational approach. In: Polycystic Ovary Syndrome, Dunaif A,
Givens JR, Haseltine F (Eds.). Cambridge, England: Blackwell Science, 1992. pp. 377-84.

7. Azziz R, Carmina E, Dewailly D, DiamantiKandarakis E, Escobar-Morreale HF, Futterweit W, et al. Positions statement: criteria for defining polycystic ovary syndrome as a predominantly hyperandrogenic syndrome: an Androgen Excess Society guideline. J Clin Endocrinol Metab. 2006; 91(11):4237-45.

8. Rotterdam, ESHRE/ASRM-Sponsored, PCOS, Consensus, Workshop, Group. Revised 2003 consensus on diagnostic criteria and long-term health risks related to polycystic ovary syndrome. Fertil Steril. 2004; 81(1):19-25

9. Zore T, Joshi NV, Lizneva D, Azziz R. Polycystic Ovarian Syndrome: Long-Term Health Consequences. Sem Reproduct Med. 2017;35 (3):271-81.

10. Dunaif A. Insulin Resistance and the polycystic ovary syndrome: Mechanism and implication for pathogenesis. Endocrine Review. 1997;18(6):774800.

11. Espinos-Gomez JJ, Corcoy R, Calaf J. Prevalence and predictors of abnormal glucose metabolism in Mediterranean women with polycystic ovary syndrome. Gynecol Endocrinol. 2009;25(3):199-204.

12. Trinder P. Determination of blood glucose using the oxidase - peroxidase system with a non-carcinogenic chromogen. J Clin Pathol. 1969;22(2);158-61.

13. Ismail AA, Astley P, Cawood M, Short F, Wakelin $\mathrm{K}$, Wheeler M. Testosterone assays: guidelines for the provision of a clinical chemistry service. Ann Clin Biochem.1986;23:135 - 45.

14. Standards of medical care in diabetes-2007. Diabetes Care. 2007;30(suppl 1):S4-41.

15. Yau TTL. Ng NYH, Cheung LP, Ma RCW. Polycystic ovary syndrome: a common reproductive syndrome with long-term metabolic consequences. Hong kong Med J. 2017;23(6):662-34.

16. Ehrmann DA. Polycystic ovary syndrome. N Engl J Med. 2005;352(12):1223-36.

17. Lerchmann E, Schwetze V, Giuliani A, Obermayerpietsh B. Assessment of glucose metabolism in polycystic ovary syndrome: HBA1c or fasting glucose compared with the oral glucose tolerance test as a screening method. Hum Reprod. 2013;28(9): 2537-44.

18. Barcellos CR, Rocha MP, Hayashidi SA, Nery M, Marcondes JA. Prevalence of abnormalities of glucose metabolism in patients with polycystic ovary syndrome. Ara Bras Endocrinol Metabol. 2007;51 (4):601-5.

19. Ehrmann DA, Barnes RB, Rosen-field RL, Cavaghan MK, Imperial J. Prevalence of impaired glucose tolerance and diabetes in women with polycystic ovary syndrome. Diabetes Care. 1999;22(1):141-6.

20. Alikor CA, Emem-Chioma PC. Epidemiology of diabetes and impaired fasting glucose in a rural community of Nigerian Niger Delta region. Niger J Med. 2015;24(2);114-24. 
21. Ejike CECC, Uka NK, Nwanchukwu SO. Diabetes and pre-diabetes in adult Nigerians: Prevalence, and correlations of blood glucose with measures of obesity. Afr J Biochem. 2015;9(3):55-60.

22. Lazaridou S, Dinas K, Tziomalos K. Prevalence, pathogenesis and management of prediabetes and type 2 diabetes mellitus in patients with polycystic ovary syndrome. Hormones. 2017;16(4):373-80.

23. Navarro G, Allard C, Xu W, Mauvais-Jarvis F. The role of androgens in metabolism, obesity, and diabetes in males and females. Obesity. 2015;23(4): 719-9.
24. Dumitrescu R, Mehedintu C, Briceag I, Purcarea VL, Hudita D. The polycystic ovary syndrome: An update on metabolic and hormonal mechanisms. J Med Life. 2015;8(2):142-5.

Cite this article as: Amadi C, Green KI.

Relationship between glucose homeostatic disorders and testosterone in adult Nigerian women with polycystic ovary syndrome. Int J Reprod Contracept Obstet Gynecol 2018;7:4336-41. 